Supplement of Biogeosciences, 17, 455-473, 2020

https://doi.org/10.5194/bg-17-455-2020-supplement

(c) Author(s) 2020. This work is distributed under

the Creative Commons Attribution 4.0 License.

(c) (i)

Supplement of

\title{
Zooplankton diel vertical migration and downward C flux into the oxy- gen minimum zone in the highly productive upwelling region off northern Chile
}

Pritha Tutasi and Ruben Escribano

Correspondence to: Pritha Tutasi (prithatutasi@udec.cl)

The copyright of individual parts of the supplement might differ from the CC BY 4.0 License. 
Table S1. Sampling data for Multinet tows by day (D) and at night (N) for five depth strata at three stations off northern Chile, during spring 2015.

\begin{tabular}{|c|c|c|c|c|c|c|}
\hline Station & Haul & Date & Time & Latitude & Longitude & Sampling Depth (m) \\
\hline \multirow{8}{*}{ St. T5 } & D1a & $29 / 11 / 2015$ & $9: 30$ & -20.05 & -70.53 & $0-600$ \\
\hline & $\mathrm{D} 1 \mathrm{~b}$ & $29 / 11 / 2015$ & $16: 30$ & -20.05 & -70.53 & $0-600$ \\
\hline & N1a & $30 / 11 / 2015$ & $2: 30$ & -20.05 & -70.53 & $0-600$ \\
\hline & N1b & $30 / 11 / 2015$ & $4: 30$ & -20.05 & -70.53 & $0-600$ \\
\hline & $\mathrm{D} 2 \mathrm{a}$ & $30 / 11 / 2015$ & $10: 30$ & -20.05 & -70.53 & $0-600$ \\
\hline & $\mathrm{D} 2 \mathrm{~b}$ & $30 / 11 / 2015$ & $15: 00$ & -20.05 & -70.53 & $0-600$ \\
\hline & $\mathrm{N} 2 \mathrm{a}$ & $30 / 11 / 2015$ & $22: 30$ & -20.05 & -70.53 & $0-600$ \\
\hline & $\mathrm{N} 2 \mathrm{~b}$ & $01 / 12 / 2015$ & $4: 30$ & -20.05 & -70.53 & $0-600$ \\
\hline \multirow{8}{*}{ St. T3 } & D1a & $02 / 12 / 2015$ & $14: 30$ & -20.07 & -70.25 & $0-600$ \\
\hline & $\mathrm{D} 1 \mathrm{~b}$ & $02 / 12 / 2015$ & $18: 00$ & -20.07 & -70.25 & $0-600$ \\
\hline & N1a & $03 / 12 / 2015$ & $0: 15$ & -20.07 & -70.25 & $0-600$ \\
\hline & N1b & $03 / 12 / 2015$ & $5: 00$ & -20.07 & -70.25 & $0-600$ \\
\hline & $\mathrm{D} 2 \mathrm{a}$ & $03 / 12 / 2015$ & $12: 00$ & -20.07 & -70.25 & $0-600$ \\
\hline & $\mathrm{D} 2 \mathrm{~b}$ & $03 / 12 / 2015$ & $16: 00$ & -20.07 & -70.25 & $0-600$ \\
\hline & $\mathrm{N} 2 \mathrm{a}$ & $04 / 12 / 2015$ & $23: 30$ & -20.07 & -70.25 & $0-600$ \\
\hline & $\mathrm{N} 2 \mathrm{~b}$ & $05 / 12 / 2015$ & $0: 15$ & -20.07 & -70.25 & $0-600$ \\
\hline \multirow{4}{*}{ St. L6 } & D1 & $09 / 12 / 2015$ & $10: 31$ & -29.29 & -71.36 & $0-600$ \\
\hline & N1 & $09 / 12 / 2015$ & $22: 30$ & -29.29 & -71.36 & $0-600$ \\
\hline & $\mathrm{D} 2$ & $10 / 12 / 2015$ & $8: 14$ & -29.29 & -71.36 & $0-600$ \\
\hline & N2 & $10 / 12 / 2015$ & $5: 00$ & -29.29 & -71.36 & $0-600$ \\
\hline
\end{tabular}


Table S2. Depth intervals representing distinctive microhabitats characterized by specific depth and oxygen concentration and the mean temperature $\left(\mathrm{T}^{\circ}\right)$ in each stratum.

\begin{tabular}{|c|c|c|c|c|c|c|c|c|}
\hline \multirow{2}{*}{$\begin{array}{c}\text { Depth } \\
\text { intervals }\end{array}$} & \multirow{2}{*}{$\begin{array}{c}\text { Strata } \\
\text { microhabitats }\end{array}$} & \multicolumn{2}{|c|}{ ST-T5 } & \multicolumn{2}{|l|}{ ST-T3 } & \multicolumn{2}{|c|}{ ST-L6 } & \multirow[t]{2}{*}{ Reference } \\
\hline & & $\mathbf{O}_{2}$ & $\mathbf{T}^{\circ}$ & $\mathbf{O}_{2}$ & $\mathbf{T}^{\circ}$ & $\mathbf{O}_{2}$ & $\mathbf{T}^{\circ}$ & \\
\hline $0-30$ & OX-ML & $>200 \mu \mathrm{M}$ & 20 & $>200 \mu \mathrm{M}$ & 19 & $>200 \mu \mathrm{M}$ & 14 & $\begin{array}{l}\text { Oxygenated Layer (Paulmier et } \\
\text { al., 2006). }\end{array}$ \\
\hline $30-90$ & OMZ-UB & $45 \mu \mathrm{M}$ & 16 & $45 \mu \mathrm{M}$ & 15 & $45 \mu \mathrm{M}$ & 13 & $\begin{array}{l}45 \mu \mathrm{M} \mathrm{O} \mathrm{O}_{2} \text { definite as upper } \\
\text { boundary of the OMZ (Escribano } \\
\text { et al., 2009; Hidalgo et al., 2005; } \\
\text { Escribano et al., 2004; Morales et } \\
\text { al., 1999) }\end{array}$ \\
\hline $90-150$ & OMZ-UC & $<20 \mu \mathrm{M}$ & 14 & $<20 \mu \mathrm{M}$ & 13 & $<20 \mu \mathrm{M}$ & 13 & $\begin{array}{l}\mathrm{O}_{2}<20-<1 \mu \mathrm{M} \text { defined as } \mathrm{OMZ} \\
\text { core Paulmier et al. (2006). }\end{array}$ \\
\hline $150-400$ & OMZ-LC & $1 \mu \mathrm{M} \mathrm{O}_{2}$ & 12 & $1 \mu \mathrm{M} \mathrm{O}_{2}$ & 12 & $4 \mu \mathrm{M} \mathrm{O}_{2}$ & 12 & $\begin{array}{l}\mathrm{O}_{2}>1-20 \mu \mathrm{M} \text { defined as } \mathrm{OMZ} \\
\text { core Paulmier et al. }(2006) \text {. }\end{array}$ \\
\hline $400-600$ & OMZ-LW & $\begin{array}{c}>20 \mu \mathrm{M} \\
\mathrm{O}_{2}\end{array}$ & 9 & $>20 \mu \mathrm{M} \mathrm{O}_{2}$ & 8 & $\begin{array}{l}\text { Non } \\
\text { CTD } \\
\text { data }\end{array}$ & 8 & slope break $>20 \mu \mathrm{M} \mathrm{O} 2$ \\
\hline
\end{tabular}


Table S3. Regressions equations between body area and dry weight (dw) given by Lehette and Hernandez Leon (2009) and Marcolini et al. (2015) to estimate biomass with the ZooScan Integrated System. Conversion factors used for biomass estimation for 27 taxonomic groups were extracted from Kiørboe (2013) and Childress and Nygaard (1973).

\begin{tabular}{|c|c|c|c|c|c|}
\hline Group & ACRONYM & a & $\mathbf{b}$ & Size & $\begin{array}{c}\text { Conversion factor } \\
\text { (C:DW) }\end{array}$ \\
\hline Amphipods & AM & 49.58 & 1.48 & Area & 0.345 \\
\hline Annelids & AN & 43.38 & 1.54 & Area & 0.299 \\
\hline Appendicularia & AP & $2.62 \mathrm{E}-08$ & 2.83 & L & 0.407 \\
\hline Bryozoa L. & BRY & 43.38 & 1.54 & Area & 0.432 \\
\hline Chaetognaths & $\mathrm{CH}$ & 23.45 & 1.19 & Area & 0.367 \\
\hline Ctenophora & $\mathrm{CT}$ & 43.38 & 1.54 & Area & 0.051 \\
\hline Small Sub C & $\mathrm{SC}$ & 45.25 & 1.59 & Area & 0.480 \\
\hline Large Sub C & LC & 45.25 & 1.59 & Area & 0.480 \\
\hline Acartia Sub C & $A C$ & 45.25 & 1.59 & Area & 0.480 \\
\hline Eucalanidae C & $\mathrm{EC}$ & 76.71 & 0.63 & Area & 0.480 \\
\hline Decapods & $\mathrm{DE}$ & 49.58 & 1.48 & Area & 0.419 \\
\hline Euphausiids & EU & 49.58 & 1.48 & Area & 0.419 \\
\hline Fish Egg & $\mathrm{FE}$ & $1.40 \mathrm{E}-10$ & 1 & Volume & 0.407 \\
\hline Foraminifera & $\mathrm{FO}$ & 89 & 1 & Volume & 0.407 \\
\hline Gastropods & $\mathrm{GA}$ & 43.38 & 1.54 & Area & 0.227 \\
\hline Hydrozoa & $\mathrm{HY}$ & 43.17 & 1.02 & Area & 0.132 \\
\hline Ichthyoplankton & IC & 43.38 & 1.54 & Area & 0.438 \\
\hline Decapoda L & $\mathrm{DL}$ & 43.38 & 1.54 & Area & 0.419 \\
\hline Echinoderm L & EL & 43.38 & 1.54 & Area & 0.407 \\
\hline Nauplius L & $\mathrm{NL}$ & 43.38 & 1.54 & Area & 0.407 \\
\hline Cirripedia L & $\mathrm{CL}$ & 43.38 & 1.54 & Area & 0.407 \\
\hline Ostracoda & OS & 99.46 & 1.28 & Area & 0.369 \\
\hline Platyhelminthes & PL & 43.38 & 1.54 & Area & 0.407 \\
\hline Pteropoda & PT & 43.38 & 1.54 & Area & 0.289 \\
\hline Radiolaria & RA & 43.38 & 1.54 & Area & 0.407 \\
\hline Salps sp & SA & 4.03 & 1.24 & Area & 0.109 \\
\hline Siphonophores & $\mathrm{SI}$ & 43.17 & 1.02 & Area & 0.109 \\
\hline
\end{tabular}


Table S4. Mean and Standard Deviation $\left( \pm\right.$ ) of integrated abundance (ind. $\mathrm{m}^{-2} \mathrm{~d}^{-1}$ ) by taxonomic groups sorted in this study during daytime/ night condition at three stations off northern Chile, during spring 2015.

\begin{tabular}{|c|c|c|c|c|c|c|c|c|c|}
\hline \multirow[b]{2}{*}{ TAXA } & \multicolumn{3}{|c|}{ T5 } & \multicolumn{3}{|c|}{ T3 } & \multicolumn{3}{|c|}{ L6 } \\
\hline & $\begin{array}{c}\text { Abundance } \\
0-600\end{array}$ & & SD & $\begin{array}{c}\text { Abundance } \\
0-600\end{array}$ & & SD & $\begin{array}{c}\text { Abundance } \\
0-600\end{array}$ & & SD \\
\hline \multicolumn{10}{|l|}{ Copepods } \\
\hline Small Copepods & 180698 & \pm & 44460 & 94735 & \pm & 24291 & 230268 & \pm & 46919 \\
\hline Large Copepods & 11384 & \pm & 2813 & 5961 & \pm & 1327 & 26840 & \pm & 4536 \\
\hline Acartia Copepod & 3 & \pm & 1 & 0 & \pm & 0 & 200 & \pm & 83 \\
\hline Eucalanidae Copepod & 3 & \pm & 1 & 20 & \pm & 6 & 44 & \pm & 9 \\
\hline Euphausiids & 434 & \pm & 82 & 341 & \pm & 66 & 1683 & \pm & 473 \\
\hline Decapoda Larvae & 428 & \pm & 132 & 292 & \pm & 62 & 400 & \pm & 88 \\
\hline Chaetognaths & 972 & \pm & 228 & 843 & \pm & 191 & 4755 & \pm & 1038 \\
\hline Annelids & 3110 & \pm & 462 & 2824 & \pm & 422 & 7395 & \pm & 847 \\
\hline Fish Eggs & 4304 & \pm & 672 & 5759 & \pm & 1020 & 20848 & \pm & 4618 \\
\hline Nauplius larvae & 1720 & \pm & 462 & 1014 & \pm & 333 & 27837 & \pm & 9587 \\
\hline Appendicularian & 10924 & \pm & 3208 & 5646 & \pm & 2098 & 11034 & \pm & 3568 \\
\hline Salps & 995 & \pm & 218 & 3193 & \pm & 817 & 10347 & \pm & 3219 \\
\hline Ostracods & 1342 & \pm & 234 & 2819 & \pm & 561 & 9899 & \pm & 1199 \\
\hline Siphonophores & 3649 & \pm & 1243 & 2487 & \pm & 823 & 4616 & \pm & 1183 \\
\hline Hydrozoan & 746 & \pm & 150 & 525 & \pm & 94 & 4397 & \pm & 1401 \\
\hline Foraminiphers & 128 & \pm & 9 & 94 & \pm & 13 & 3564 & \pm & 370 \\
\hline Gastropods & 450 & \pm & 115 & 180 & \pm & 24 & 1806 & \pm & 435 \\
\hline Platyhelminthes & 0 & \pm & 0 & 0 & \pm & 0 & 2410 & \pm & 1054 \\
\hline Cirripedia Larvae & 112 & \pm & 28 & 58 & \pm & 11 & 2190 & \pm & 839 \\
\hline Bryozoan & 113 & \pm & 29 & 63 & \pm & 15 & 298 & \pm & 120 \\
\hline Amphipods & 44 & \pm & 9 & 195 & \pm & 41 & 116 & \pm & 27 \\
\hline Pteropods & 152 & \pm & 64 & 19 & \pm & 5 & 135 & \pm & 39 \\
\hline Ctenophores & 1 & \pm & 0 & 14 & \pm & 6 & 119 & \pm & 38 \\
\hline Echinoderm larvae & 7 & \pm & 3 & 0 & \pm & 0 & 18 & \pm & 8 \\
\hline Radiolarian & 1 & \pm & 0 & 4 & \pm & 2 & 14 & \pm & 5 \\
\hline Decapods & 11 & \pm & 3 & 0 & \pm & 0 & 0 & \pm & 0 \\
\hline Ichthyoplankton & 4 & \pm & 1 & 1 & \pm & 0 & 0 & \pm & 0 \\
\hline Total ind. $\mathrm{m}^{2}$ & 221735 & \pm & 53698 & 127085 & \pm & 31229 & 371235 & \pm & 76618 \\
\hline
\end{tabular}


Table S5- Daily average of relative abundance (\%) for the 27 zooplankton groups sorted in this study during daytime/ night condition at three stations off northern Chile, during spring 2015. Each depth stratum represents a specific oxygen condition.

\begin{tabular}{|c|c|c|c|c|c|c|c|c|c|c|c|c|c|c|c|c|c|c|}
\hline \multirow{4}{*}{ TAXA } & \multirow{4}{*}{$\begin{array}{l}\text { OX } \\
\text { ML }\end{array}$} & \multicolumn{4}{|c|}{ St. T5 } & \multirow{4}{*}{ Total } & \multirow{5}{*}{$\begin{array}{l}\text { OX } \\
\text { ML }\end{array}$} & \multirow{2}{*}{\multicolumn{4}{|c|}{$\frac{\text { St. T3 }}{\text { OMZ }}$}} & \multirow{3}{*}{ Total } & \multirow{5}{*}{$\begin{array}{l}\text { OX } \\
\text { ML }\end{array}$} & \multirow{2}{*}{\multicolumn{4}{|c|}{$\frac{\text { St. L6 }}{\text { OMZ }}$}} & \multirow{3}{*}{ Total } \\
\hline & & \multicolumn{4}{|c|}{ OMZ } & & & & & & & & & & & & & \\
\hline & & UB & $\mathrm{UC}$ & $\mathrm{LC}$ & LW & & & UB & UC & LC & LW & & & UB & $\mathrm{UC}$ & LC & LW & \\
\hline & & & & & & & & Majo & Group & & & & & & & & & \\
\hline COPEPODS & & & & & & 87 & & & & & & 79 & & & & & & 69 \\
\hline SC & 46 & 27 & 4 & 1 & 2 & 81 & 47 & 17 & 8 & 1 & 2 & 75 & 32 & 17 & 5 & 3 & 4 & 62 \\
\hline LC & 3 & 2 & $<1$ & $<1$ & $<1$ & 5 & 3 & 1 & $<1$ & $<1$ & $<1$ & 5 & 3 & 3 & 1 & $<1$ & 1 & 7 \\
\hline $\mathbf{A C}$ & 0 & $<1$ & 0 & 0 & 0 & $<1$ & 0 & 0 & 0 & 0 & 0 & 0 & $<1$ & 0 & $<1$ & 0 & 0 & $<1$ \\
\hline EC & 0 & 0 & 0 & $<1$ & $<1$ & $<1$ & 0 & $<1$ & 0 & 0 & $<1$ & $<1$ & $<1$ & 0 & $<1$ & $<1$ & 0 & $<1$ \\
\hline FE & 1 & $<1$ & $<1$ & $<1$ & $<1$ & 2 & 2 & 1 & $<1$ & 1 & $<1$ & 5 & 2 & 3 & $<1$ & $<1$ & $<1$ & 6 \\
\hline NL & $<1$ & $<1$ & $<1$ & $<1$ & $<1$ & $<1$ & $<1$ & $<1$ & $<1$ & $<1$ & $<1$ & $<1$ & 6 & 1 & $<1$ & $<1$ & $<1$ & 7 \\
\hline AP & 3 & 1 & $<1$ & $<1$ & $<1$ & 5 & 4 & $<1$ & $<1$ & $<1$ & $<1$ & 4 & 2 & 1 & $<1$ & $<1$ & $<1$ & 3 \\
\hline OTHERS & 3 & 2 & 1 & 1 & $<1$ & $<6$ & 5 & 3 & 1 & 1 & $<1$ & 11 & 6 & 6 & 1 & 1 & 1 & 15 \\
\hline
\end{tabular}


Table S6- Carbon respiration $\left(\mathrm{mg} \mathrm{C} \mathrm{m} \mathrm{m}^{-2}\right)$ in the Oxygen minimum zone (90-600 $\mathrm{m}$ ) by zooplankton groups sorted in this study during daytime/ night condition at three stations off northern Chile, during spring 2015. Respiration rate was estimated using regression provided by Ikeda (1985).

\begin{tabular}{lrrr}
\hline \multicolumn{1}{c}{ Taxa } & St. T5 & \multicolumn{1}{c}{ St. T3 } & \multicolumn{1}{c}{ St. L6 } \\
\hline Amphipods & 0.06 & 0.16 & 0.68 \\
Annelids & 4.77 & 7.23 & 13.09 \\
Appendicularia & 0.00 & 0.00 & 0.00 \\
Bryozoan & 0.00 & 0.00 & 0.00 \\
Chaetognaths & 0.62 & 0.58 & 6.72 \\
Cirripedia L & 0.00 & 0.02 & 0.04 \\
Ctenophores & 0.05 & & 0.08 \\
Acartia C & & & 0.03 \\
Eucalanidae C & 0.01 & 0.09 & 0.48 \\
Large C & 7.28 & 7.23 & 38.94 \\
Small C & 12.14 & 12.29 & 21.27 \\
Decapods & 2.63 & & \\
Decapods L & 0.21 & & \\
Euphausiids & 6.51 & 7.17 & 33.41 \\
Fish Egg & 0.00 & 0.00 & 0.00 \\
Foraminiphers & 0.06 & 0.06 & 0.62 \\
Gastropods & 0.01 & 0.04 & 0.14 \\
Hydrozaa & 0.34 & 1.18 & 3.58 \\
Ichthyoplankton & 0.07 & 0.40 & \\
Nauplius L & 0.02 & 0.02 & 0.24 \\
Ostracods & 1.61 & 2.59 & 15.57 \\
Platyhelminthes & & & 0.83 \\
Pteropods & 0.00 & 0.00 & 0.00 \\
Radiolarian & 0.00 & 0.02 & 0.03 \\
Salps & 0.14 & 0.45 & 0.24 \\
Siphonophores & 0.16 & 0.17 & 0.75 \\
\hline
\end{tabular}


Table S7- Migrant biomass ( $\mathrm{mg} \mathrm{C} \mathrm{m}^{-2}$ ) for taxonomic groups of zooplankton sampled in the deep stratum (90-600 m) at northern Chile at 3 stations: off Iquique (Stations T5 and T3) and off Coquimbo (Station L6) during the austral spring 2015. SD= Standard deviation of the migrant biomass estimated from $\mathrm{n}=4$ (St. T5 and St. T3) and $n=2$ (St. L6).

\begin{tabular}{|c|c|c|c|c|c|c|c|c|c|}
\hline \multirow[b]{2}{*}{ TAXA } & \multicolumn{3}{|c|}{ T5 } & \multicolumn{3}{|c|}{ T3 } & \multicolumn{3}{|c|}{ L6 } \\
\hline & MB & & SD & MB & & SD & MB & & SD \\
\hline Decapoda_L & 3.7 & \pm & 3.5 & 446.7 & \pm & 257.9 & 847.6 & \pm & 485.5 \\
\hline Euphausiids & 389.1 & \pm & 214.6 & 330.0 & \pm & 158.4 & 123.7 & \pm & 96.7 \\
\hline Large C & 17.3 & \pm & 11.6 & 4.3 & \pm & 3.0 & 157.5 & \pm & 110.5 \\
\hline Small C & 18.4 & \pm & 10.8 & 25.3 & \pm & 13.5 & 31.0 & \pm & 53.3 \\
\hline Eucalanid C & 0.3 & \pm & 0.2 & 3.3 & \pm & 1.9 & 14.4 & \pm & 12.4 \\
\hline Acartia C & 0.0 & \pm & 0.0 & 0.0 & \pm & 0.0 & 0.0 & \pm & 0.0 \\
\hline Annelida & 8.6 & \pm & 15.9 & 31.1 & \pm & 39.4 & 83.4 & \pm & 57.5 \\
\hline Ichthyoplankton & 4.9 & \pm & 7.7 & 50.1 & \pm & 28.9 & 0.0 & \pm & 0.0 \\
\hline Ostracoda & 3.3 & \pm & 2.2 & 8.5 & \pm & 4.7 & 42.9 & \pm & 41.9 \\
\hline Chaetognaths & 35.5 & \pm & 20.1 & 0.9 & \pm & 0.9 & 3.8 & \pm & 21.2 \\
\hline Hydrozaa & 3.2 & \pm & 2.0 & 4.8 & \pm & 3.0 & 23.3 & \pm & 16.2 \\
\hline Amphipoda & 6.5 & \pm & 3.9 & 7.5 & \pm & 1.6 & 8.4 & \pm & 8.0 \\
\hline Salps & 1.4 & \pm & 0.4 & 11.7 & \pm & 6.6 & 0.1 & \pm & 0.2 \\
\hline Decapods & 7.8 & \pm & 4.5 & 0.0 & \pm & 0.0 & 0.0 & \pm & 0.0 \\
\hline Ctenophora & 0.9 & \pm & 0.5 & 0.0 & \pm & 0.0 & 3.7 & \pm & 2.1 \\
\hline Siphonophores & 2.2 & \pm & 1.1 & 0.0 & \pm & 0.1 & 1.6 & \pm & 0.6 \\
\hline Platyhelminthes & 0.0 & \pm & 0.0 & 0.0 & \pm & 0.0 & 1.9 & \pm & 1.1 \\
\hline Foraminifera & 0.3 & \pm & 0.2 & 0.2 & \pm & 0.1 & 0.8 & \pm & 2.7 \\
\hline Radiolaria & 0.0 & \pm & 0.0 & 0.1 & \pm & 0.1 & 1.2 & \pm & 0.6 \\
\hline Gastropoda & 0.1 & \pm & 0.0 & 0.1 & \pm & 0.0 & 0.8 & \pm & 0.6 \\
\hline Nauplius_L & 0.0 & \pm & 0.1 & 0.1 & \pm & 0.0 & 0.6 & \pm & 0.7 \\
\hline Cirripedia_L & 0.0 & \pm & 0.0 & 0.0 & \pm & 0.0 & 0.5 & \pm & 0.1 \\
\hline Pteropoda & 0.0 & \pm & 0.0 & 0.0 & \pm & 0.0 & 0.0 & \pm & 0.0 \\
\hline Appendicularia & 0.0 & \pm & 0.0 & 0.0 & \pm & 0.0 & 0.0 & \pm & 0.0 \\
\hline Fish_Egg & 0.0 & \pm & 0.0 & 0.0 & \pm & 0.0 & 0.0 & \pm & 0.0 \\
\hline Echinoderm_L & 0.0 & \pm & 0.0 & 0.0 & \pm & 0.0 & 0.0 & \pm & 0.0 \\
\hline Bryozoa & 0.0 & \pm & 0.0 & 0.0 & \pm & 0.0 & 0.0 & \pm & 0.0 \\
\hline
\end{tabular}

